\title{
Die neue eidgenössische Prüfung hat einen Mehrwert
}

\author{
Sissel Guttormsen ${ }^{a}$, André P. Perruchoud \\ ${ }^{a}$ Prof. Dr. phil., Direktorin, Institut für Medizinische Lehre, Universität Bern; b Prof. Dr. med., Präsident der eidgenössischen Prüfungskommission für Humanmedizin
}

Wir danken Herrn Professor Martin Fey für die ausführliche Reaktion [1] auf unseren Artikel [2] und die Hinweise auf wichtige, nicht gelöste Problemkreise. Zwischen zwei lateinischen Redewendungen vermittelt Herr Fey seine Botschaft mit dem Tenor, dass früher alles besser war. Es ist uns ein Anliegen, die Notwendigkeit des Fortschritts in Bezug auf Inhalt, Form und Wissenschaft für die eidgenössische Prüfung (EP) darzustellen. Als Erstes einige Richtigstellungen:

- Nicht die eidgenössische Prüfungskommission (PK) hat die Inhalte der EP oder den Wechsel von der alten zur neuen Ordnung beschlossen, sondern das Medizinalberufegesetz (MedBG) legt die Rahmenbedingungen für die Prüfungen der Medizinalberufe fest. Die PK setzt die EP im Einklang mit dem Gesetz um und bezieht aktiv die verschiedenen Stakeholder, insbesondere die medizinischen Fakultäten und deren Experten, ein.

- Die neue EP besteht seit 5 Jahren aus zwei klinischen Prüfungen: eine schriftliche und eine praktische. Bekanntlich setzt das «Tun» eine Wissensbasis voraus. Ein duales Verständnis, wie Herr Fey den Unterschied zwischen «Wissen» und «Tun» aufstellt, liegt uns fern. Die EP erfasst klinisches Können auf zwei Arten: schriftlich und praktisch. Bei beiden Teil-Prüfungen ist Clinical Reasoning eine Voraussetzung für erfolgreiche Lösungen. Die statistischen Analysen zeigen, dass sich die zwei aktuellen Prüfungsformen gut ergänzen und unterschiedliche Aspekte prüfen.

- Nach sechs Jahren Studium ist die EP eine letzte Qualitätssicherungsmassnahme für die Lizenzierung und damit den Eintritt in die klinische Arbeit. Selbstverständlich wird angestrebt, dass möglichst alle Kandidatinnen und Kandidaten die Prüfung bestehen. Bei jenen, die nicht bestehen, handelt es sich um eine negative Selektion einer Minderheit. Diese nach abgeschlossenem Medizinstudium zu erkennen, muss auf methodisch und wissenschaftlich stichfester Grundlage beruhen.

- Rekurse kommen immer wieder vor, sie sind aber selten und sie leiten mit Sicherheit nicht unser Handeln. Im Vordergrund steht ein valides und faires Examen mit vergleichbaren Bedingungen für alle!
Herr Fey vermisst die alten Zeiten, in denen der direkten Interaktion zwischen Meister und Lehrling mehr Raum gegeben wurde. Selbstverständlich ist die direkte Interaktion des erfahrenen Lehrers mit den Studierenden und realen Patienten zentral für die medizinische Ausbildung und sollte so oft wie möglich im Rahmen des sechsjährigen Medizinstudiums umgesetzt werden. Es steht jeder Fakultät frei, während der Ausbildung unterschiedliche Lehr- und Assessmentformen umzusetzen, vieles in der Art ist auch bereits erfolgreich

\section{Im Vordergrund steht ein valides und}

faires Examen mit vergleichbaren Bedingungen für alle!

umgesetzt worden (z.B. MiniCEX und DOPS). Die Lizenzierungsprüfung nach Abschluss des Studiums kann eindeutig nicht das richtige Setting für unstrukturierte Expertenprüfungen bieten, dies hat mehrere Gründe:

- Machbarkeit: Die Zahl der Kandidierenden, die an der EP antreten, steigt glücklicherweise Jahr für Jahr, 2015 circa 900. Eine Expertenprüfung nach Modell Fey würde sehr viele Patienten und Experten in Anspruch nehmen. Was wäre, wenn während weniger Tage pro Fakultät mehrere Hundert echte Patienten zum Einsatz kommen müssten? Oder müsste die Prüfung über mehrere Wochen abgehalten werden? Wie sollen die Kliniken, insbesondere die Bettenstationen mit ihrem hektischen klinischen Alltag, die Rekrutierung und Vorbereitung der vielen benötigten Patienten, unter Berücksichtigung der methodischen Mindestanforderungen, bewältigen können?

- Transparenz: Eine inhaltlich relevante und methodisch korrekte Prüfung basiert auf einer fachlichen und quantitativ ausbalancierten Aufstellung der inhaltlichen Ziele der Prüfung (Blueprint). Der Blueprint wird den Studierenden kommuniziert. Es ist kaum machbar, einen Blueprint für die EP mit passenden echten Patienten planbar abzubilden. Nicht zufällig vorhandene Patienten sollen bestimmen, was geprüft wird, sondern eine ausgewogene Themenauswahl. Damit sollen genügend Wissen und Fertigkeiten in mehreren Bereichen sichergestellt werden. 
- Fairness: In der alten Prüfung stellten sich notgedrungen sehr unterschiedliche Patienten zur Verfügung, der eine leidet an einer Aphasie, der andere teilt seine Diagnose gleich mit. Es soll für die Ausbildungsstufe adäquat geprüft werden, losgelöst von der Komplexität der vorhandenen Patienten. Zudem wird bei der EP durch die hohe Anzahl der Prüfenden pro Kandidat (12 Prüfer) sichergestellt, dass eine möglichst objektive Gesamtbeurteilung entsteht. Auch die Prüfer sind nicht alle so uneingeschränkt objektiv, wie Herr Fey es sich vorstellt. Jeder hält sich so weit an die Vorgaben, wie er das für notwendig findet, Tagesform und Empathie spielen eine Rolle in der direkten Interaktion.

\section{Nicht zufällig vorhandene Patienten} sollen bestimmen, was geprüft wird, sondern eine ausgewogene Themenauswahl.

- Gesamtes Spektrum: Beim Einsatz von echten Patienten in einer nationalen Prüfungssituation würden auch ethische und praktische Bedenken einige $\mathrm{Pa}$ tientengruppen ausschliessen (z.B. Notfälle, Psychiatrie). Durch gut trainierte standardisierte Patienten kann hingegen ein breites Spektrum von Fällen und Situationen abgedeckt werden.

Hat die EP einen Mehrwert? Wir sind überzeugt, dass die wichtigsten Vorzüge der EP im nationalen Austausch und durch Spiegelung des geforderten integrierten Wissens am Schluss des Studiums liegen. Wie bei allen Prüfungen bewirkt auch die EP einen unbestrittenen Druck zum Lernen. Für die Fakultäten gibt die EP wertvolle objektive Feedbacks über die Lehre. Daraus leiten sich Anstösse zu Verbesserungen wie auch Informationen über das, was gut läuft, ab. Die weiteren Auswirkungen der EP und die wissenschaftlichen Hintergründe haben wir anderweitig publiziert [3].
Die Reformen des Medizinstudiums und der Prüfungen basieren auf fachlichen, wissenschaftlichen, aber auch gesellschaftlichen Überlegungen. Die prädiktive Validität einer solchen lizenzierenden Prüfung wurde von deren Pendants in den USA und Kanada hinsichtlich einiger Aspekte bereits gezeigt $[4,5]$. Solche Studien sind sehr aufwendig und für die EP noch nicht verfügbar. Die EP ist entsprechend dem aktuellen Wissensstand für solche lizenzierenden Prüfungen aufgebaut. So wie sich Kliniker an den Stand der Forschung in ihren Fachgebieten halten müssen, so können wir uns auch in der Lehre und bei Prüfungen nicht dem Stand der Forschung und dem Fortschritt verschliessen, das sind wir unseren Studierenden und der Gesellschaft schuldig.

Warum Herr Fey und andere mit ihm, die sich sonst für Wissenschaft in ihren Fachgebieten stark machen, Prüfungen anders behandeln wollen, bleibt offen. Aber es macht Sinn, wenn Herr Fey die neue EP in Frage stellt. Nur anhand von kritischen Stimmen kann man eine solide Reflexion über das Aktuelle und das Zukünftige führen. Kritische Stimmen sind notwendig, damit man mit Vorsicht, Mass und Vernunft der Innovation Platz macht.

Literatur

1 Fey M. Annuntio vobis gaudium magnum - ein neues klinisches Staatsexamen! Schweiz Ärztezeitung. 2016;97(4):153-4.

2 Guttormsen S, Perruchoud AP. Pionierarbeit für die Abschlussprüfung Medizin. Schweiz Ärztezeitung. 2015;96(50-51):1846-8.

3 Guttormsen S, Beyeler C, Bonvin R, Feller S, Schirlo C, Schnabel K, et al. The new licencing examination for human medicine: from concept to implementation. Swiss Med Wkly. 2013;143:w13897.

4 Tamblyn R, Abrahamowicz M, Dauphinee WD, Hanley JA, Norcini J, Girard N, et al. Association between licensure examination scores and practice in primary care. JAMA. 288(23):3019-26.

5 Norcini JJ, Boulet JR, Opalek A, Dauphinee WD. The relationship between licensing examination performance and the outcomes of care by international medical school graduates. Acad Med. 2014;89(8):1157-62. 